\title{
Japanese epidemiological survey with consensus statement on Japanese guidelines for treatment of iron overload in bone marrow failure syndromes
}

\author{
Takahiro Suzuki - Masao Tomonaga - Yasushi Miyazaki · \\ Shinji Nakao · Kazuma Ohyashiki · Itaru Matsumura • \\ Yutaka Kohgo $\cdot$ Yoshiro Niitsu $\cdot$ Seiji Kojima $\cdot$ Keiya Ozawa
}

Received: 30 April 2008/Accepted: 2 June 2008/Published online: 27 June 2008

(C) The Japanese Society of Hematology 2008

\begin{abstract}
Many patients with bone marrow failure syndromes need frequent transfusions of red blood cells, and most of them eventually suffer from organ dysfunction induced by excessively accumulated iron. The only way to treat transfusion-induced iron overload is iron chelating therapy. However, most patients have not been treated effectively because daily/ continuous administration of deferoxamine is difficult for outpatients. Recently, a novel oral iron chelator, deferasirox, has been developed, and introduction of the drug may help many patients benefit from iron chelation therapy. In this review, we will discuss the current status of iron overload in transfusion-dependent patients, and the development of Japanese guidelines for the treatment of iron overload in Japan, which were established by the National Research Group on Idiopathic Bone Marrow Failure Syndromes in Japan.
\end{abstract}

Keywords Bone marrow failure syndrome .

Iron overload · Iron chelation · Guidelines

T. Suzuki $\cdot$ K. Ozawa $(\bowtie)$

Division of Hematology, Department of Medicine,

Jichi Medical University, 3311-1 Yakushiji,

Shimotsuke-shi, Tochigi 329-0498, Japan

e-mail: kozawa@ms2.jichi.ac.jp

M. Tomonaga $\cdot$ Y. Miyazaki

Department of Hematology and Molecular Medicine Unit,

Atomic Bomb Disease Institute, Nagasaki University Graduate

School of Biomedical Sciences, Nagasaki, Japan

S. Nakao

Department of Cellular Transplantation Biology,

Kanazawa University Graduate School

of Medical Science, Kanazawa, Japan

K. Ohyashiki

First Department of Internal Medicine (Department

of Hematology), Tokyo Medical University, Tokyo, Japan

\section{Introduction}

Many patients with aplastic anemia (AA) or myelodysplastic syndromes (MDS) need frequent transfusions of red blood cells (RBCs). One unit (derived from $200 \mathrm{~mL}$ of whole blood) of RBC transfusion in Japan contains about $100 \mathrm{mg}$ of iron. Because there is no physiological mechanism for iron excretion in humans, and daily iron excretion is no more than $1 \mathrm{mg}$ in a healthy man, repeated $\mathrm{RBC}$ transfusions will soon result in iron overload. Excess iron is mainly deposited in the liver, heart and pancreas, and causes organ dysfunction $[1,2]$.

As phlebotomy is not an option because of the underlying bone marrow failure, the only way to treat iron overload is by iron chelation therapy. However, difficulty in optimal administration of deferoxamine (DFO, Desfer$\mathrm{al}^{\circledR}$ ) in Japan has hampered effective chelation, and currently most patients are not treated effectively [3].

\author{
I. Matsumura \\ Department of Hematology and Oncology, \\ Osaka University Graduate School of Medicine, \\ Osaka, Japan \\ Y. Kohgo \\ Department of Medicine, Division of Gastroenterology \\ and Hematology/Oncology, Asahikawa Medical College, \\ Asahikawa, Japan \\ Y. Niitsu \\ Fourth Department of Internal Medicine, \\ Sapporo Medical University School of Medicine, \\ Sapporo, Japan \\ S. Kojima \\ Department of Paediatrics, \\ Nagoya University Graduate School of Medicine, \\ Nagoya, Japan
}


Recently, a novel oral iron chelator, deferasirox (Exjade ${ }^{\circledR}$ ), has been introduced in more than 60 countries, including Japan. The introduction of deferasirox may improve compliance with iron chelation therapy [4]. Under these circumstances, the National Research Group on Idiopathic Bone Marrow Failure Syndromes in Japan drew up Japanese guidelines for the treatment of transfusion-induced iron overload. Herein, we describe the current status of iron overload in transfusion-dependent patients in Japan, and development of the proposed guidelines for the treatment of transfusion-induced iron overload.

\section{Current status of transfusion-induced iron overload in Japan}

In 2005, the first nationwide survey on iron overload in transfusion-dependent patients in Japan was carried out [3]. This retrospective survey investigated the outcomes of iron overload-related morbidity and mortality from August 2001 to December 2005. A questionnaire was sent to hematology departments in hospitals all over Japan, and 43 hospitals responded by returning data on 292 patients.

Demographic data showed that MDS and AA accounted for about $80 \%$ of the underlying diseases: MDS, $52.1 \%$; AA, 30.8\%; pure red cell aplasia (PRCA), $5.1 \%$; and myelofibrosis (MF), $4.5 \%$. Serum ferritin levels were significantly correlated with the lifetime total number of RBC transfusion units received. Figure 1 shows the relationship between the number of RBC units and mean ferritin level, indicating the percentage of patients with an abnormal ferritin level $(\geq 1,000 \mathrm{ng} / \mathrm{mL})$ for any total number of RBC units received as analyzed by a logistics model. The goodness-of-fit of this model between theoretical and actual values was assessed by Pearson chi-squared test, and the estimated number of RBC units required to raise ferritin to $\geq 1,000 \mathrm{ng} / \mathrm{mL}$ in 50 and $75 \%$ of patients was calculated as 21.5 and 43.4 units, respectively.

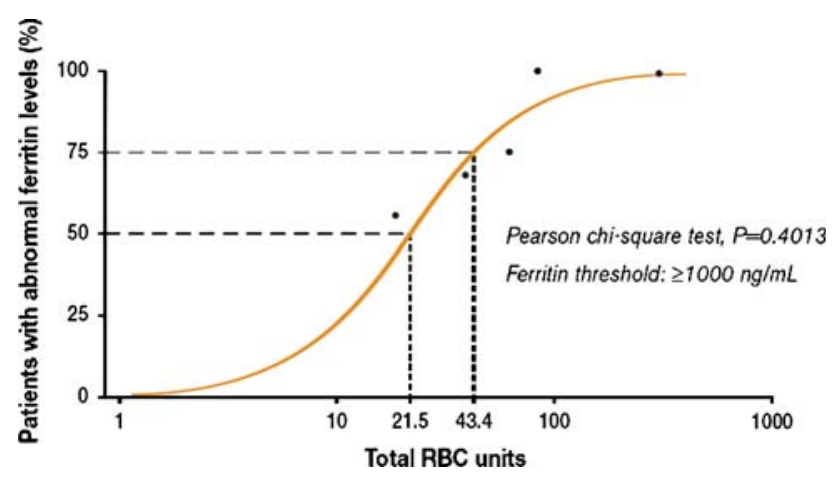

Fig. 1 Relationship between serum ferritin and total number of red blood cell units. [3] Modified with permission from Takatoku et al. Eur J Haematol. 2007;78:487-494. ${ }^{\circledR} 2007$ Blackwell Publishing
Serum glutamic oxaloacetic transaminase (SGOT) and serum glutamic pyruvic transaminase (SGPT) abnormalities were significantly correlated with transfusion frequency and increased ferritin levels; there was a significantly $(P<0.0001)$ higher prevalence of SGOT and SGPT abnormality in patients with high serum ferritin than in those whose serum ferritin was $<1,000 \mathrm{ng} / \mathrm{mL}$ (Fig. 2). Moreover, among patients in whom cardiac function was evaluated, abnormalities were found in $21.9 \%$, and cardiac abnormality was weakly correlated with serum ferritin levels. These data indicate that ferritin levels can be a useful predictor of hepatic and cardiac dysfunction. Fasting blood sugar (FBS) abnormality was also correlated with transfusion frequency.

In the survey, 75 deaths were reported, most of which were caused by infection and leukemia. However, cardiac and hepatic failure was noted in $24 \%$ and $6.7 \%$ of cases, respectively. Patients who died from cardiac or hepatic failure had received more transfusions than those who died from other causes, and among 38 patients in whom serum ferritin levels were available, 37 patients died with serum ferritin levels $\geq 1,000 \mathrm{ng} / \mathrm{mL}$; the majority of patients ( 24 patients) had serum ferritin levels $>5,000 \mathrm{ng} / \mathrm{mL}$. These data indicate that multiple transfusion therapy is associated with a high risk of fatal complications caused by iron overload. Recently, similar analyses have been reported describing that transfusion-dependent MDS patients show significantly shorter survival than those who do not require transfusions and that transfusion-induced iron overload significantly affects survival [5].

\section{Iron chelation therapy}

As phlebotomy is not an option because of underlying bone marrow failure, the only way to treat iron overload is with iron chelation therapy. Until recently, the only available iron chelating agent in Japan was DFO. Because of the limited absorption from the gastrointestinal tract and short biological half-life of the agent, the drug must be administered by parenteral injections at least 5-7 times a week, or continuously for optimal effectiveness [6]. In the survey, $43.2 \%$ of patients received DFO, but only $8.6 \%$ received DFO daily or continuously; most of the patients were administered the drug intermittently (average once per 1.9 weeks) or concurrently with transfusion [3]. While improvements in serum ferritin, SGOT, SGPT and FBS were noted in the patients who received DFO daily or continuously, these data did not improve, and rather worsened, in those without optimal administration (Table 1). This indicates that appropriate administration of the chelating agent is needed for sufficient therapeutic results. 
Fig. 2 Relationship between serum transaminase abnormality and serum ferritin levels. [3] Modified with permission from Takatoku et al. Eur J Haematol. 2007;78:487-494. ${ }^{\odot} 2007$ Blackwell Publishing

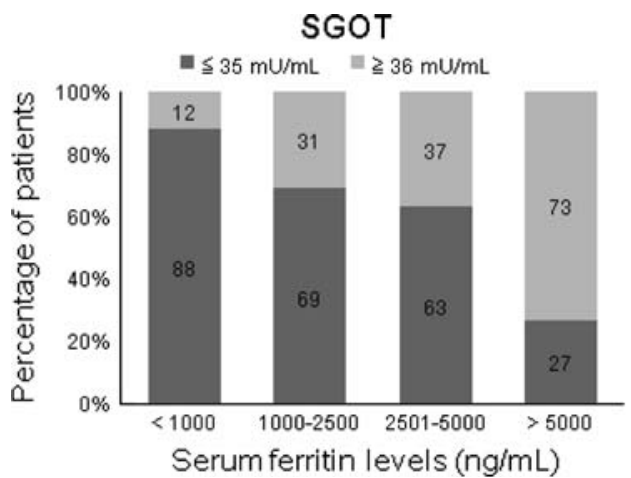

Table 1 Average changes in laboratory values during the period of transfusion dependence in patients receiving deferoxamine treatment

\begin{tabular}{lcrr}
\hline Parameter & Intermittent (once/1.9 week) & Concurrent with transfusion & Daily/continuous \\
\hline Serum ferritin $^{\mathrm{a}, \mathrm{b}}(\mathrm{ng} / \mathrm{mL})$ & $+2222.8(n=36)$ & $+2204.8(n=19)$ & $-1135.2(n=9)$ \\
SGOT $^{\mathrm{a}, \mathrm{c}}(\mathrm{mU} / \mathrm{mL})$ & $+28.0(n=53)$ & $+40.0(n=30)$ & $-9.2(n=10)$ \\
SGPT $(\mathrm{mU} / \mathrm{mL})$ & $+28.6(n=53)$ & $+10.3(n=30)$ & $-28.8(n=10)$ \\
FBS $(\mathrm{mg} / \mathrm{dL})$ & $+31.2(n=31)$ & $+8.2(n=12)$ & $-4.8(n=5)$ \\
\hline
\end{tabular}

[3] Modified with permission from Takatoku et al. Eur J Haematol. 2007;78:487-494. ${ }^{\circ} 2007$ Blackwell Publishing

${ }^{a}$ Intermittent versus continuous, $P<0.05$

${ }^{\text {b }}$ Continuous versus concurrent, $P<0.01$

${ }^{\mathrm{c}}$ Continuous versus concurrent, $P<0.05$

Moreover, it has also been reported that iron chelation not only reduced iron burden and improved organ dysfunction, but also ameliorated the hemoglobin levels of iron-overloaded patients [7, 8]. Although the biological mechanism of the hematopoietic recovery remains to be elucidated, this fact indicates that iron itself negatively impacts on hematopoiesis, and in some conditions removal of iron burden from the hematopoietic environment can restore normal hematopoiesis.

Deferasirox is easily absorbed in the gastrointestinal tract and has an elimination half-life of $8-16 \mathrm{~h}$, which means that deferasirox is continuously present in the plasma with oncedaily dosing [9]. In a large Phase III trial, deferasirox was comparable with DFO at decreasing iron burden in $\beta$-thalassemic patients [10]. Deferasirox also reduced iron burden in patients with various anemias including MDS [11]. These findings indicate that oral iron chelators can improve patients' quality of life by ameliorating organ dysfunction and preventing iron damage, even improving hematopoiesis itself. Oral iron chelators are expected to prolong survival of transfusion-dependent patients.

\section{Japanese guidelines for the treatment of iron overload in transfusion-dependent patients}

The clinical significance of iron chelation is undeniable and requires attention. With the availability of deferasirox in
Japan, the frequency of continuous treatment may be strengthened and many more patients can benefit from chelation therapy. To help optimal iron chelation therapy, the National Research Group on Idiopathic Bone Marrow Failure Syndromes drew up the Japanese guidelines for the treatment of transfusion-induced iron overload. To date, guidelines for iron overload have been developed in several countries [6, 12-14], and the Japanese guidelines were designed to align with the international guidelines (see the paper by Dr. Gattermann in this issue). The essential features of the Japanese guidelines are depicted in Fig. 3 and Table 2 .

The contents of the guidelines are as follows:

Patients who may benefit from chelation therapy: The guidelines are applicable to transfusion-dependent patients with primary (MDS, AA, PRCA, MF, etc.) and secondary (chemotherapy-induced, etc.) bone marrow failure. Transfusion-dependent patients are defined as those receiving $>2$ $\mathrm{RBC}$ units/month for $\geq 6$ months. Because organ dysfunction becomes symptomatic after a certain period of time, it is suggested that iron chelation therapy is offered to patients with an expected survival of more than 1 year. The international guidelines for MDS patients also recommend that they should have a life expectancy of $\geq 1$ year.

Diagnosis of iron overload: After patients become transfusion dependent, regular examination of serum ferritin is required to monitor iron burden at least once every 3 months. For early diagnosis of organ dysfunction, 
Fig. 3 A flow chart for the treatment of transfusiondependent iron overload

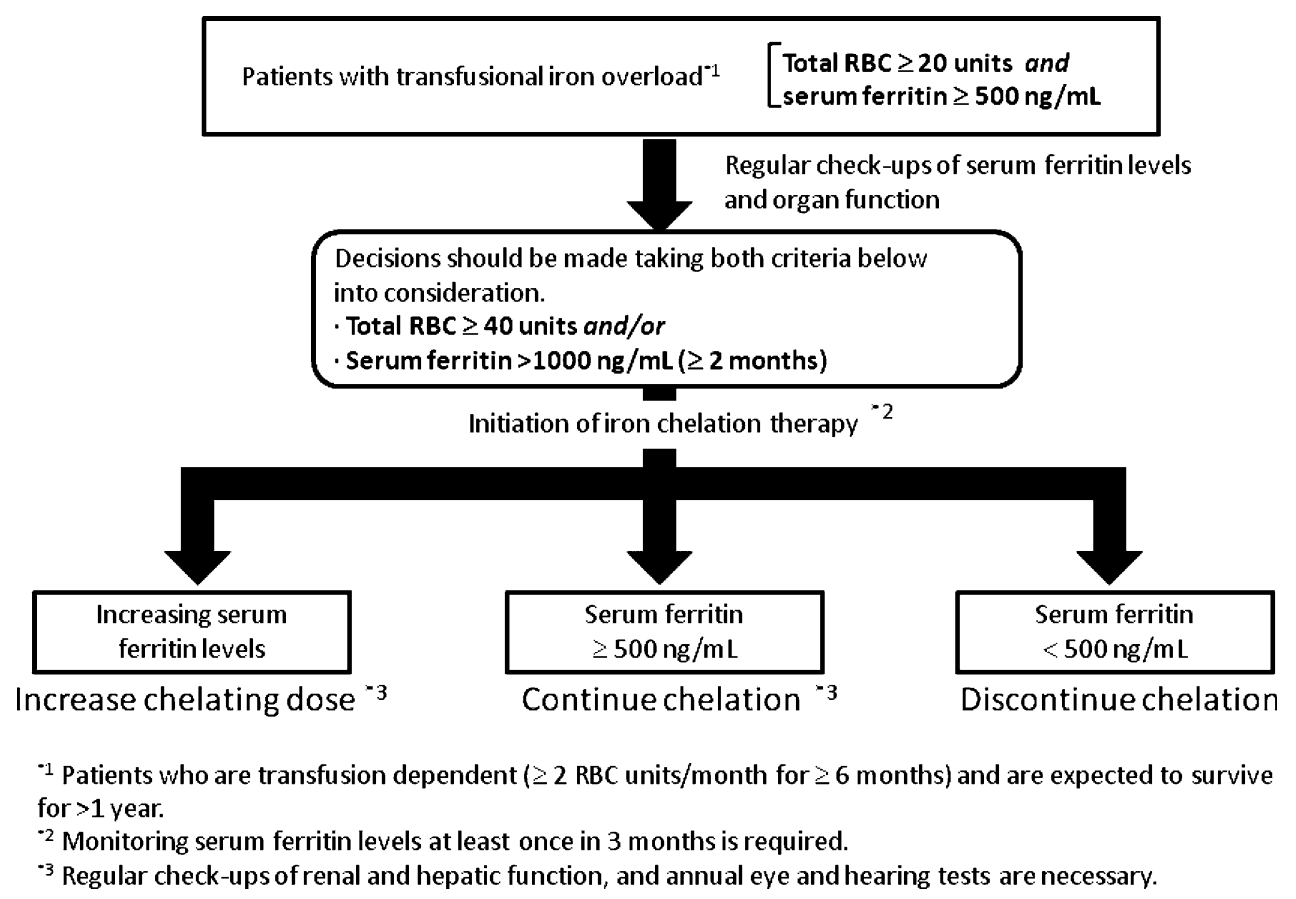

Regular check-ups of serum ferritin levels and organ function

Decisions should be made taking both criteria below consideration.

Total RBC $\geq \mathbf{4 0}$ units and/or

Initiation of iron chelation therapy ${ }^{*} 2$ periodic check-ups of cardiac, hepatic and pancreatic endocrine functions are recommended.

Patients can be said to be iron overloaded when their serum ferritin levels reach $>500 \mathrm{ng} / \mathrm{mL}$ and when they have received $>20$ Japanese $\mathrm{RBC}$ units (in pediatric patients, $>50 \mathrm{~mL} / \mathrm{kg}$ body weight). Severity of iron overload is determined by serum ferritin levels and organ dysfunction (Table 2, lower part).

Initiating iron chelation therapy: Administration of an iron chelator is the only recommended treatment for iron overload in patients with bone marrow failure. To initiate iron chelation therapy, confirmation of serum ferritin levels $>1,000 \mathrm{ng} / \mathrm{mL}$ for more than 2 months, at least in two successive examinations, is recommended. The nationwide survey reported that more than $90 \%$ of patients who suffered from organ dysfunction had serum ferritin levels $>1,000 \mathrm{ng} / \mathrm{mL}$, and prevalence of hepatic dysfunction increases in parallel with ferritin levels [3] (Fig. 2). Therefore, a serum ferritin level $>1,000 \mathrm{ng} / \mathrm{mL}$ is considered the appropriate point to initiate iron chelation. However, serum ferritin levels are not reliable in patients with inflammatory conditions such as Still's disease and hemophagocytic syndrome, or in those with malignancies. In these cases, transfusion history should be taken into account. Therefore, receiving a total of more than 40 Japanese RBC transfusion units (in pediatric patients, $>100 \mathrm{~mL} / \mathrm{kg}$ body weight) was included as another recommended criterion. As mentioned previously, about $75 \%$ of patients who received $>40 \mathrm{RBC}$ units have serum ferritin levels $>1,000 \mathrm{ng} / \mathrm{mL}$, indicating that 40 units of RBC transfusion can be a good indicator of transfusion-induced hyperferritinemia. However, transfusion history alone is also not reliable, because serum ferritin levels may not increase in patients with chronic bleeding and hemolysis. Furthermore, patients who have already discontinued transfusion therapy with successful treatment may not require iron chelation therapy. If neither of these two criteria is applicable, chelation therapy should not be started.

Target ferritin maintenance levels and adverse effects of iron chelators: During chelation therapy, monitoring of iron burden and organ functions should be continued. After initiating chelation therapy, serum ferritin levels should decrease, but if they continue to increase, even 3-6 months after starting treatment, an increase in dose is necessary. When patients are minimally transfusion dependent $(<2$ RBC units/month) or already free of transfusions, dose adjustment must be determined carefully.

It is recommended that serum ferritin levels are maintained at $500-1,000 \mathrm{ng} / \mathrm{mL}$, and when ferritin levels are below $500 \mathrm{ng} / \mathrm{mL}$ at two successive examinations, chelators should be discontinued. As an excessive reduction in iron burden is harmful, the guidelines have determined this target value $(500-1,000 \mathrm{ng} / \mathrm{mL})$ with a safety margin.

As iron chelating agents can induce adverse effects on the kidney, liver and sensory organs [10], regular examination of renal and hepatic functions, and periodical (prior to treatment and annually after initiation) ophthalmologic examinations and hearing tests, are recommended. If an abnormal increase in serum creatinine level is noticed, the drug should be decreased or discontinued. In patients with a high risk of renal dysfunction, weekly monitoring of creatinine level is recommended, at least during the first 
Table 2 Japanese guidelines for transfusional iron overload (main points)

\begin{tabular}{|c|c|c|}
\hline Patients & \multicolumn{2}{|c|}{ Transfusion-dependent patients with bone marrow failure syndromes who are likely to survive for $>1$ year } \\
\hline Diagnosis of iron overload & \multicolumn{2}{|c|}{$\begin{array}{l}\text { 1. Total } \mathrm{RBC}>20 \text { units }^{\mathrm{a}} \text { (in pediatric patients, } \mathrm{RBCs}>50 \mathrm{~mL} / \mathrm{kg} \text { body weight) } \\
\text { and } \\
\text { 2. Serum ferritin }>500 \mathrm{ng} / \mathrm{mL}\end{array}$} \\
\hline $\begin{array}{l}\text { Criteria for initiating chelation } \\
\text { therapy }\end{array}$ & $\begin{array}{l}\text { 1. Total } \mathrm{RBC}>40 \text { units }^{\mathrm{a}} \text { (in pediatric patients, RBCs } \\
\text { and/or } \\
\text { 2. Serum ferritin }>1,000 \mathrm{ng} / \mathrm{mL} \\
\text { Decisions should be made taking both criteria into co } \\
\text {-with chronic bleeding or hemolysis; } \\
\text {-who no longer need } \mathrm{RBC} \text { transfusions; } \\
\text {-with complications that chronically raise serum ferriti } \\
\text { hemophagocytic syndrome and malignancies }\end{array}$ & usion; e.g., Still's disease, \\
\hline $\begin{array}{l}\text { Target serum ferritin } \\
\text { maintenance level }\end{array}$ & \multicolumn{2}{|l|}{ Serum ferritin $500-1,000 \mathrm{ng} / \mathrm{mL}$} \\
\hline \multicolumn{3}{|c|}{ Classified severity of iron overload } \\
\hline Serum ferritin $(\mathrm{ng} / \mathrm{mL})$ & With normal organ function & With organ dysfunction \\
\hline$>500$ & Stage $1 \mathrm{~A}$ & Stage 1B \\
\hline$>1,000$ & Stage $2 \mathrm{~A}$ & Stage $2 \mathrm{~B}$ \\
\hline$>2,500$ & Stage $3 \mathrm{~A}$ & Stage $3 \mathrm{~B}$ \\
\hline$>5,000$ & Stage $4 \mathrm{~A}$ & Stage 4B \\
\hline
\end{tabular}

The severity of iron overload is defined by serum ferritin level and organ dysfunction (cardiac, liver and pancreatic endocrine dysfunction). The dysfunction must be considered to be related to iron overload; i.e., the organ dysfunction progresses as serum ferritin or transfusion burden increase

The criteria for specific organ dysfunction are as follows

-Cardiac dysfunction: LVEF $<50 \%$

-Hepatic dysfunction: abnormal transaminase levels, fibrosis and cirrhosis of the liver

-Pancreatic endocrine dysfunction: impaired glucose tolerance

a 20 and 40 units of the Japanese RBC transfusion correspond to 10 and 20 Western RBC units, respectively

month. Furthermore, if drug-induced hepatic injury is suspected, withdrawal of the drug with appropriate treatments is needed. It has been reported that iron chelators can cause hearing loss and cataracts. Therefore, if any signs of dysfunction are noticed a dose reduction or discontinuation of the drug is necessary and prompt consultation by an ophthalmologist or otorhinolaryngologist is required. In pediatric patients, annual monitoring of height, weight and state of secondary sex characteristics are needed for an early diagnosis of abnormal development.

\section{Conclusions}

The retrospective survey of transfusion-dependent patients revealed that the mortality rate is raised in heavily ironoverloaded patients, with liver and cardiac dysfunction being the primary cause of death [3]. Daily or continuous chelation therapy is effective in reducing iron burden and improving organ function, but practically, daily or continuous administration through parenteral injection is difficult.
In Japan, a novel oral chelator, deferasirox, has recently been approved. Oral iron chelators can improve compliance of treatment and many more patients who need iron chelation may benefit from a reduction in iron burden and improvement of organ function, which ultimately may lead to the improvement of patients' prognosis and quality of life.

Acknowledgments This work was supported by a grant (Research on Intractable Diseases) from the Ministry of Health, Labor and Welfare of Japan. The authors thank Dr. Norbert Gattermann for his valuable advice in establishing the guidelines.

\section{References}

1. Kushner JP, Porter J, Olivieri N. Secondary iron overload. Hematology/American Society of Hematology Education Program Book: American Society of Hematology; 2001.

2. McLaren GD, Muir WA, Kellermeyer RW. Iron overload disorders: natural history, pathogenesis, diagnosis, and therapy. Crit Rev Clin Lab Sci. 1983;19:205-66.

3. Takatoku M, Uchiyama T, Okamoto $S$, et al. Retrospective nationwide survey of Japanese patients with transfusion- 
dependent MDS and aplastic anemia highlights the negative impact of iron overload on morbidity/mortality. Eur J Haematol. 2007;78:487-94.

4. Shashaty G, Frankewich R, Chakraborti T, et al. Deferasirox for the treatment of chronic iron overload in transfusional hemosiderosis. Oncology (Williston Park). 2006;20:1799-806, 1811; discussion 1811-3, 1817.

5. Malcovati L, Porta MG, Pascutto C, et al. Prognostic factors and life expectancy in myelodysplastic syndromes classified according to WHO criteria: a basis for clinical decision making. J Clin Oncol. 2005;23:7594-603.

6. Gattermann N, Porter J, Lopes L, Seymour J. Consensus statement on iron overload in myelodysplastic syndromes. Hematol Oncol Clin North Am. 2005;19:18-25.

7. Di Tucci AA, Murru R, Alberti D, Rabault B, Deplano S, Angelucci E. Correction of anemia in a transfusion-dependent patient with primary myelofibrosis receiving iron chelation therapy with deferasirox (Exjade, ICL670). Eur J Haematol. 2007;78:540-42.

8. Jensen PD, Heickendorff L, Pedersen B, et al. The effect of iron chelation on haemopoiesis in MDS patients with transfusional iron overload. Br J Haematol. 1996;94:288-99.
9. Piga A, Galanello R, Forni GL, et al. Randomized phase II trial of deferasirox (Exjade, ICL670), a once-daily, orally-administered iron chelator, in comparison to deferoxamine in thalassemia patients with transfusional iron overload. Haematologica. 2006;91:873-80

10. Cappellini MD, Cohen A, Piga A, et al. A phase 3 study of deferasirox (ICL670), a once-daily oral iron chelator, in patients with beta-thalassemia. Blood. 2006;107:3455-62.

11. Porter J, Vichinsky E, Rose C, et al. A phase II study with ICL670 (Exjade), a once-daily oral iron chelator, in patients with various transfusion-dependent anemias and iron overload. Blood. 2004;104:abstract 3193.

12. Alessandrino EP, Amadori S, Barosi G, et al. Evidence- and consensus-based practice guidelines for the therapy of primary myelodysplastic syndromes. A statement from the Italian Society of Hematology. Haematologica. 2002;87:1286-306.

13. Bowen D, Culligan D, Jowitt $\mathrm{S}$, et al. Guidelines for the diagnosis and therapy of adult myelodysplastic syndromes. Br J Haematol. 2003;120:187-200.

14. Greenberg PL, Baer MR, Bennett JM, et al. Myelodysplastic syndromes clinical practice guidelines in oncology. J Natl Compr Canc Netw. 2006;4:58-77. 